Ingeniare. Revista chilena de ingeniería, vol. $18 \mathrm{~N}^{\circ} 1,2010$, pp. 105-119

\title{
SIMULACIÓN BIDIMENSIONAL DE UN SISTEMA DE COMBUSTIÓN INESTABLE
}

\section{TWO-DIMENSIONAL SIMULATION OF AN UNSTABLE COMBUSTION SYSTEM}

\author{
Javier Achury Varila $^{1} \quad$ Carlos Duque Daza $^{1} \quad$ Carlos Galeano Urueña $^{1}$ \\ Recibido 16 de marzo de 2009, Aceptado 15 de enero de 2010 \\ Received: March 16, 2009 Accepted: January 15, 2010
}

\begin{abstract}
RESUMEN
La inestabilidad en la combustión es una condición indeseada en algunos sistemas de combustión como en turbinas de gas por ejemplo. Se refiere a la presencia autogenerada de oscilaciones en la presión que pueden afectar a la cámara de combustión y de paso llegar a generar ruido. Una reciente tendencia generalizada en los procesos de combustión apunta al uso de mezclas pobres para la reducción de contaminantes, no obstante que este tipo de mezclas son más susceptibles a la inestabilidad en la combustión. Las complicadas relaciones que gobiernan el fenómeno se pueden resumir como el acoplamiento entre la llama y la acústica del sistema. En el presente trabajo se presenta un planteamiento numérico que permite aproximarse al fenómeno a través de la solución de un modelo de combustión básico implementado computacionalmente. En este modelo se simula una autoexcitación del sistema a través de oscilaciones en la entrada de flujos de reactantes. Finalmente, se comparan los resultados de la simulación numérica con otras simulaciones y datos experimentales.
\end{abstract}

Palabras clave: Inestabilidad en combustión, función de transferencia de llama, simulación numérica directa.

\begin{abstract}
The Combustion instability is an undesirable condition reached in some combustion systems, as during the operation of gas turbines. It refers to self-excited oscillations of pressure that may affect the combustion chamber and generate noise. A recent generalized tendency in combustion processes aims to the use of lean combustion (low fuel/air ratios) for pollutants reduction, nevertheless this sort of mixtures are more susceptible to combustion instabilities. The complex relationship that generates the phenomenon can be summarized as the coupling between flame and acoustics. In this paper it is outlined a numerical approach to this phenomenon by solving a basic computational combustion model (by Direct Numerical Simulation). In this model a self-excited system is simulated through imposed oscillations in reactants flows. Finally, results for this numerical simulation are compared against other simulations and experimental available data.
\end{abstract}

Keywords: Combustion instabilities, flame transfer function, direct numerical simulation.

\section{INTRODUCCIÓN}

La inestabilidad en la combustión es un fenómeno termoacústico que se describe a través del criterio de Rayleigh [1,2]. Este fenómeno se puede definir como el acoplamiento inestable entre una tasa de calor fluctuante entregada por la combustión y las ondas acústicas (ver Figura 1) en un sistema de combustión particular [3,4]. Una fuente inestable de calor, sea cual sea ésta, produce ondas acústicas $p^{\prime}(\vec{x}, t)$ que se propagan por el medio afectando a la misma tasa de calor fluctuante $q^{\prime}(\vec{x}, t)$, formando así un ciclo cerrado. Según el criterio de Rayleigh, este ciclo tendrá un crecimiento positivo si estas tasas $p^{\prime}(\vec{x}, t)$ y $q^{\prime}(\vec{x}, t)$ se encuentran en fase, de forma tal que perturbaciones infinitesimales en el fluido se pueden ver amplificadas, en particular las ondas sonoras [3,5]. Una de las formas matemáticas más ampliamente usada para expresar este criterio es:

$$
R_{e}=\int_{0}^{T} q^{\prime}(t) p^{\prime}(t) d t
$$

Donde $T$ es el periodo de la oscilación y $R_{e}$ es precisamente el índice de Rayleigh. Esta relación revela la importancia de la fase existente entre las ondas $q^{\prime}$ y $p^{\prime}$ en la amplificación o atenuación del fenómeno (índice de Rayleigh positivo o negativo).

\footnotetext{
1 Ingeniería Mecánica y Mecatrónica. Universidad Nacional de Colombia. Kr. 30 45-03, Edif.453-401. Bogotá, Colombia. E-mail: jachuryv@ unal.edu.co; caduqued@unal.edu.co; chgaleanou@unal.edu.co
} 


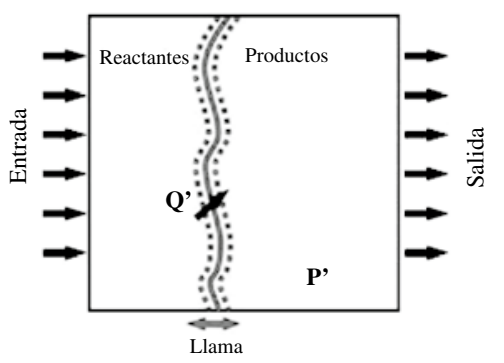

Figura 1. Representación esquemática del fenómeno de inestabilidad en la combustión.

Existen actualmente dos líneas o metodologías principales para intentar predecir o simular la estabilidad o inestabilidad característica de los dispositivos de combustión, inclusive dentro de su etapa de diseño $[6,7]$. La primera de ellas se centra en hacer un tratamiento de las ecuaciones para un flujo reactante con el fin de obtener una ecuación termoacústica de onda, en la cual se vincula la tasa fluctuante de calor entregado por la combustión a una ecuación acústica. En este tipo de metodologías de análisis termoacústicos, como la presentada en la referencia [8], es común encontrar la ecuación de onda para flujos reactantes expresada como:

$$
\nabla \cdot\left(c_{o}^{-2} \nabla p_{1}\right)-\frac{\partial^{2} p_{1}}{\partial t^{2}}=(\gamma-1) \frac{\partial \dot{q}^{\prime}}{\partial t}
$$

Donde $\bar{c}_{o}$ es la velocidad promedio del sonido, $p_{1}$ es la componente fluctuante de la presión y $\gamma$ es la relación de calores específicos $c_{p} / c_{v}$, siendo $c_{p}$ el calor específico a presión constante y $c_{v}$ el calor específico del gas a volumen constante. Esta ecuación gobierna el campo de presiones acústicas y se diferencia de la ecuación de onda para flujos no reactantes, o ecuación de Helmholtz, por el término no homogéneo $(\gamma-1) \frac{\partial \dot{q}^{\prime}}{\partial t}$.

Con el fin de plantear una solución a esta ecuación, es necesario incluir una segunda expresión para la tasa de calor fluctuante $\dot{q}^{\prime}(\vec{x}, t)$, de manera que sea posible plantear un sistema cerrado de ecuaciones. Una forma común de expresar la tasa de calor es por medio de la función de transferencia de llama ${ }^{2}$, la cual según el modelo $n-\tau$, presentado en $[9,10]$, se expresa como:

$$
\frac{\dot{q}_{1}(\vec{x}, t)}{\dot{q}_{0}(\vec{x}, t)}=n(\vec{x}) \frac{\vec{u}_{1}(\vec{x}, t-\tau) \cdot \vec{n}_{r e f}}{\vec{u}_{0}(\vec{x}) \cdot \vec{n}_{r e f}}
$$

En este modelo $n$ se denomina índice de interacción, y corresponde a la amplificación normalizada de la onda de velocidad $u$ con una dirección de referencia $n_{r e f}$,

2 FTF por sus siglas en inglés. desde una posición de referencia $\left(x_{r e f}, y_{\text {ref }}\right)$. Igualmente, en esta expresión $\tau$ es denominado tiempo de retardo, y corresponde a un tiempo característico de desfase entre las ondas indicadas en la ecuación (3), en la que el subíndice $O$ hace referencia al valor medio de la variable, en tanto que el subíndice 1 al valor fluctuante. El uso de esta expresión hace el método semiempírico, ya que las tasas fluctuantes de calor son parte de la solución del problema de combustión inestable y no son conocidas a priori, excepto experimentalmente o a través de simulación. Esta función de transferencia de llama en sí misma constituye una característica del sistema de combustión, y se usa para evitar descripciones complejas de las geometrías, los modelos de turbulencia, los métodos de solución, entre otros elementos, y en vez de ello establecer una relación directa entre la excitación del sistema y su respuesta en términos de la fluctuación de la tasa de calor entregada por la combustión (Figura 2).

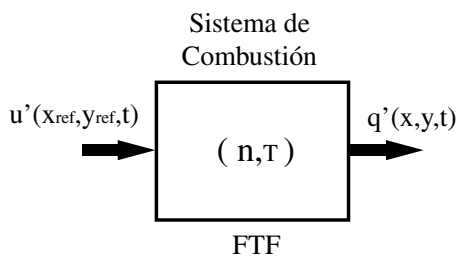

Figura 2. Función de transferencia de llama (FTF) para un sistema de combustión.

Una segunda línea de investigación se enfoca en hacer un tratamiento numérico directo del flujo a través de las ecuaciones de Navier-Stokes [2, 11, 12], en general basado en potentes, pero matemáticamente costosas, herramientas de aproximación, las cuales acoplan al modelo ecuaciones de combustión. Un ejemplo de este tipo de herramientas usadas en aproximaciones de la combustión inestable, por mencionar uno relevante, es el método LES (Large-Eddy Simulations) [2, 10, 13-16]. Las formulaciones varían según los diferentes grados de complejidad con los que se aborda el problema, es decir, de la forma en la que se haga el tratamiento de la química y tasa de reacción de la combustión, el transporte molecular o el manejo de la compresibilidad del flujo, entre otros aspectos. Sin embargo, la selección de la aproximación numérica a realizar dependerá del balance entre exactitud y costo computacional requerido [17].

El presente trabajo se desarrolló en el marco de esta última tendencia de tratamiento computacional, siguiendo como principal premisa la obtención de un modelo numérico de moderada complejidad y relativo bajo costo computacional, capaz de capturar la naturaleza del fenómeno de acoplamiento entre la inestabilidad de la combustión y los perfiles de presión acústica. En la primera sección de este artículo se presenta el modelo 
físico del fenómeno, así como el modelo matemático implementado, mostrando las ecuaciones de trabajo, las correspondientes aproximaciones numéricas y las condiciones de frontera utilizadas. Posteriormente se presentan y discuten los resultados de un caso de aplicación del modelo construido, para finalmente ofrecer las principales conclusiones respecto de los beneficios e implicaciones de la metodología desarrollada.

\section{FENÓMENO Y MODELO MATEMÁTICO}

Como se mencionó anteriormente, una de las principales premisas de este trabajo era la obtención de un modelo numérico de moderada complejidad, buena exactitud y relativo bajo costo computacional, que pudiese capturar la naturaleza del fenómeno de acoplamiento entre la inestabilidad de la combustión y los perfiles de presión acústica, sin el uso de exigentes máquinas de cómputo. Para este fin, el problema se definió como la aproximación numérica al efecto de la combustión inestable de un gas compresible, fluyendo en una cámara de combustión modelada con la geometría del dominio bidimensional mostrado en la Figura 3, como una representación simplificada de las características de una cámara genérica de una turbina a gas.

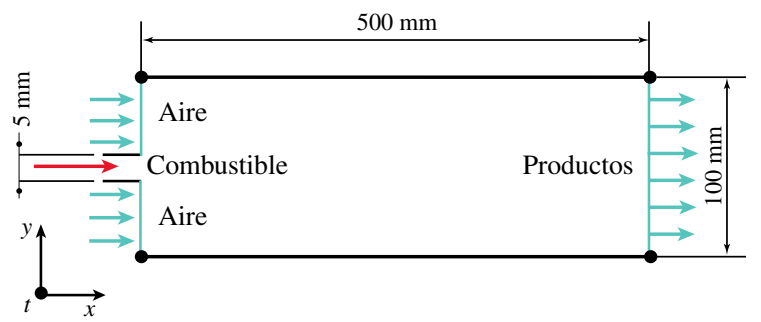

Figura 3. Dimensiones espaciales del dominio de la simulación.

El fenómeno se delimitó mediante la implementación de un modelo de dinámica de gases reactantes basado en una simplificación de las ecuaciones de Navier-Stokes y en un modelo de combustión de llama no-premezclada. El modelo matemático para el flujo de gases se basó en el conjunto de ecuaciones de Euler (continuidad, momento, energía) más una ecuación de estado para un gas no viscoso y no turbulento sometido a un proceso de combustión de llama no-premezclada. El modelo de combustión utilizado para la simulación de la llama no-premezclada se derivó de la ecuación de conservación de la fracción de mezcla con una tasa de reacción instantánea y de química tabulada.

Estructura de una llama por difusión (no-premezcla) La estructura simplificada de una llama permite afirmar que esta es una zona donde ocurre una reacción química en la que los reactantes se convierten en productos y se tienen cambios en las propiedades del fluido debido a una adición de calor en una pequeña interfase o zona de reacción, como se pretende representar en la Figura 4. En llamas difusivas o deflagrantes, los flujos de reactantes se encuentran inicialmente separados y la reacción de combustión se da en la interfase combustible-oxidante, lugar donde se tiene tanto la mezcla como la reacción [18]. Si una llama se desplaza inestablemente alrededor de una zona media se producen efectos de combustión inestable.

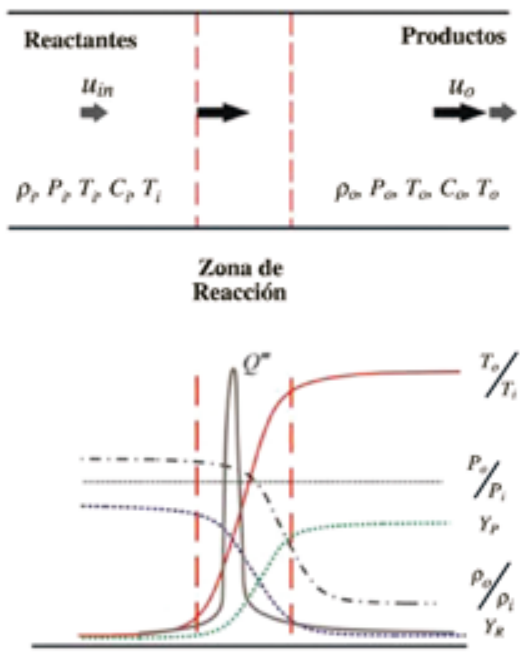

Figura 4. Características y variación de propiedades a través de una llama de deflagración.

\section{Modelo de Combustión}

Cada uno de los diferentes estudios llevados a cabo acerca de sistemas de combustión inestable, por ejemplo en las referencias [11,17], tienen como característica el uso de un modelo particular de combustión. Parte del presente trabajo consiste en mostrar la aplicación de un modelo de combustión sin premezcla basado en tasas de reacción instantáneas y de equilibrio químico, gobernado por la ecuación de conservación de la fracción de mezcla $f$ $[13,16,19,20]$. Bajo las consideraciones implícitas de tal modelo de combustión, este es relativamente simple frente a otros, dado que está basado en la solución de una ecuación de difusión para la fracción de mezcla $(f)$ y a la reconstrucción de los productos de equilibrio de la reacción y la temperatura a partir de esta variable. Es decir, como producto del modelo, se puede contar con las funciones para la concentración molar de las especies $\chi_{i}=\chi_{i}(f)$ (química tabulada) y para la temperatura $T=T(f)$.

Si se definen las condiciones de frontera de un sistema de combustión para este modelo, se tiene que, en los puntos donde se considera un flujo de combustible, la fracción de mezcla adquiere el valor de $f=1$, mientras que en los puntos donde fluye el oxidante $f=0$, formándose un 
proceso de mezcla difusiva dominado por la ecuación de conservación de $f$. De acuerdo con este modelo, dentro del dominio del problema, la fracción de mezcla tomará valores $0 \leq f \leq 1$, definiéndose así las zonas de reacción y formación de productos. Esta formulación de combustión permite un modelado relativamente sencillo para la descripción de la formación de especies fuentes de polución $\left(N O_{x}\right.$ principalmente), razón por la que es usada con relativa frecuencia en la simulación de cámaras de combustión de turbinas a gas $[16,19-21]$.

Para reconstruir los productos de combustión de equilibrio, con una mezcla particular, existen diversos códigos computacionales $[18,21]$. En el presente trabajo se hizo uso del preprocesador del paquete Fluent denominado predPDF. En la Figura 5 se muestran las concentraciones de las especies consideradas como una función de la fracción de mezcla, obtenidas haciendo uso de este paquete.

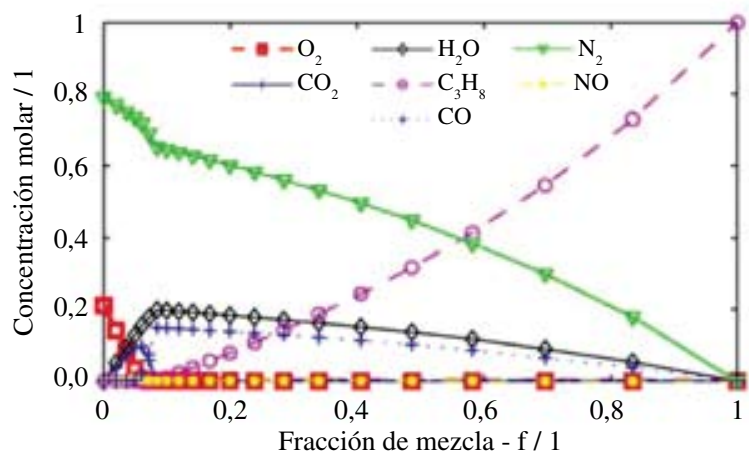

Figura 5. Composición molar de especies según la fracción de mezcla $\left(\chi_{i}=\chi_{i}(f)\right)$, para equilibrio químico.

\section{Sistema Base}

El conjunto de ecuaciones básicas de trabajo se deriva del modelo para un flujo bidimensional de un gas no viscoso, no turbulento y en el que no se considera ningún tipo de transferencia de calor, bien sea por radiación o conducción, a través de las paredes o del mismo gas. Este conjunto de ecuaciones consiste en una simplificación de las ecuaciones de continuidad y momento de NavierStokes en las direcciones longitudinal $(x)$ y transversal (y). Adicionalmente, se acopla a este sistema la ecuación de conservación de $f$ (que se denominará aquí como ecuación de combustión). En resumen, el modelo matemático utilizado se describe de acuerdo con el sistema de ecuaciones diferenciales parciales mostrado en (4) $[16,22]$ :

$$
\begin{aligned}
& \frac{\partial \rho}{\partial t}+\frac{\partial(\rho u)}{\partial x}+\frac{\partial(\rho v)}{\partial y}=0 \\
& \frac{\partial(\rho u)}{\partial t}+\frac{\partial\left(\rho u^{2}+p\right)}{\partial x}+\frac{\partial(\rho u v)}{\partial y}=0 \\
& \frac{\partial(\rho v)}{\partial t}+\frac{\partial\left(\rho v^{2}+p\right)}{\partial y}+\frac{\partial(\rho u v)}{\partial x}=0 \\
& \frac{\partial(\rho f)}{\partial t}+\frac{\partial(\rho u f)}{\partial x}+\frac{\partial(\rho v f)}{\partial y}-\frac{\partial}{\partial x}\left(\rho D \frac{\partial f}{\partial x}\right)-\frac{\partial}{\partial y}\left(\rho D \frac{\partial f}{\partial y}\right)=0
\end{aligned}
$$

Donde $\rho$ es la densidad del fluido, $u$ es la velocidad en la dirección $x$ (según el sistema de referencia definido en la Figura 3), $v$ es la velocidad en la dirección $y, p$ es la presión, $D$ es el coeficiente de difusión de la mezcla y $f$ es la fracción de mezcla. Además, para la inclusión de la ecuación de energía se desprecian los efectos de conducción de calor en el gas, de manera que esta relación de conservación se puede enunciar como:

$$
\frac{\partial(\rho E)}{\partial t}+\frac{\partial(\rho u E+p u)}{\partial x}+\frac{\partial(\rho v E+p v)}{\partial y}=\dot{q}
$$

A través de (5) se afirma que el cambio de energía del sistema depende del calor añadido por la combustión, siendo esta la cantidad $q$. Asimismo, $E$ representa la energía específica total del sistema (energía interna $e$, cinética $u^{2} / 2+v^{2} / 2$ y potencial), la cual, despreciando los cambios en la energía potencial, se puede expresar como:

$$
\begin{gathered}
E=e+\frac{u^{2}}{2}+\frac{v^{2}}{2} \\
e=c_{v} T
\end{gathered}
$$

En este punto es importante recordar que para un gas ideal la energía interna es función esencialmente de la temperatura $T, e=e(T)$. Incorporando la relación de calores específicos $\gamma$, la ecuación de estado del gas se puede expresar tal como se muestra en (8), para un gas ideal:

$$
\begin{aligned}
& p=\rho R T \\
& R=c_{p}-c_{v}=(\gamma-1) c_{v} \\
& p=(\gamma-1) \rho e
\end{aligned}
$$

Siendo $R$ la constante del gas. Así, a través de la ecuación de estado, se puede cerrar el conjunto de ecuaciones del sistema base (modelo propuesto), estableciendo una relación directa entre la energía interna (o temperatura) y la presión. Eliminando la energía interna (e) de (5) y usando de las ecuaciones de conservación de momento, se obtiene una forma de la ecuación de la energía en términos de la presión. 


$$
\frac{\partial p}{\partial t}+u \frac{\partial p}{\partial x}+v \frac{\partial p}{\partial y}+\gamma p \frac{\partial u}{\partial x}+\gamma p \frac{\partial v}{\partial y}=(\gamma-1) \dot{q}
$$

En esta expresión se hace evidente que la cantidad $(\gamma-1) \dot{q}$ (tasa de calor entregada al sistema por efecto de la combustión) es el término que vuelve el sistema no homogéneo, constituyendo la fuente que fuerza el sistema. Esta fuente, en caso de poseer una componente de oscilación, se transformará finalmente en una fuente acústica [23]. El conjunto de ecuaciones (4) y (9) constituye el sistema de ecuaciones de Euler, a partir del cual se puede derivar la ecuación de onda, mediante la descomposición de la presión y la velocidad en una componente media y otra fluctuante, $p=p_{0}+p_{1}$ y $u=u_{0}+u_{1}$, y su subsecuente linealización [24]. De esta manera el modelo planteado conserva su naturaleza termoacústica, aun cuando no se haga uso de ecuaciones acústicas o termoacústicas explícitamente, ya que se propone trabajar con modelos no linealizados. Una discusión acerca de los modelos

lineales y no-lineales para el problema de combustión inestable se puede encontrar en la referencia [25].

Adicionalmente, en el modelo matemático propuesto se efectuó un cambio de variables básicas a variables primitivas [26], lo cual permite expresar el sistema de ecuaciones únicamente en términos de las derivadas directas de dichas variables primitivas, denominadas aquí como $q_{n}$ :

$$
\left(\begin{array}{l}
q_{1} \\
q_{2} \\
q_{3} \\
q_{4} \\
q_{5}
\end{array}\right)=\left(\begin{array}{l}
\rho \\
\rho u \\
\rho v \\
p \\
\rho f
\end{array}\right)
$$

$\mathrm{Al}$ reemplazar estas variables primitivas en el sistema base (4) y (9) y desarrollando las respectivas derivadas, es posible obtener un sistema de ecuaciones en términos de variables primitivas:

$$
\begin{gathered}
\frac{\partial q_{1}}{\partial t}+\frac{\partial q_{2}}{\partial x}+\frac{\partial q_{3}}{\partial y}=0 \\
\frac{\partial q_{2}}{\partial t}+2 \frac{q_{2}}{q_{1}} \frac{\partial q_{2}}{\partial x}-\left(\frac{q_{2}}{q_{1}}\right)^{2} \frac{\partial q_{1}}{\partial x}+\frac{\partial q_{4}}{\partial x}+\frac{q_{3}}{q_{1}} \frac{\partial q_{2}}{\partial y}+\frac{q_{2}}{q_{1}} \frac{\partial q_{3}}{\partial y}-\frac{q_{2} q_{3}}{q_{1}} \frac{\partial q_{1}}{\partial y}=0 \\
\frac{\partial q_{3}}{\partial t}+2 \frac{q_{3}}{q_{1}} \frac{\partial q_{3}}{\partial y}-\left(\frac{q_{3}}{q_{1}}\right)^{2} \frac{\partial q_{1}}{\partial y}+\frac{\partial q_{4}}{\partial y}+\frac{q_{3}}{q_{1}} \frac{\partial q_{2}}{\partial x}+\frac{q_{2}}{q_{1}} \frac{\partial q_{3}}{\partial x}-\frac{q_{2} q_{3}}{q_{1}} \frac{\partial q_{1}}{\partial x}=0 \\
\frac{\partial q_{4}}{\partial t}+\frac{q_{2}}{q_{1}} \frac{\partial q_{4}}{\partial x}+\gamma \frac{q_{4}}{q_{1}} \frac{\partial q_{2}}{\partial x}-\gamma \frac{q_{2} q_{4}}{q_{2}^{2}} \frac{\partial q_{1}}{\partial x}+\frac{q_{3}}{q_{1}} \frac{\partial q_{4}}{\partial y}+\gamma \frac{q_{4}}{q_{1}} \frac{\partial q_{3}}{\partial y}-\gamma \frac{q_{3} q_{4}}{q_{1}^{2}} \frac{\partial q_{4}}{\partial y}=(\gamma-1) \dot{q} \\
\frac{\partial q_{5}}{\partial t}+\frac{q_{2}}{q_{1}} \frac{\partial q_{5}}{\partial x}+\frac{q_{5}}{q_{1}} \frac{\partial q_{2}}{\partial x}-\frac{q_{2} q_{5}}{q_{1}^{2}} \frac{\partial q_{1}}{\partial x}+\frac{q_{3}}{q_{1}} \frac{\partial q_{5}}{\partial y}+\frac{q_{5}}{q_{1}} \frac{\partial q_{3}}{\partial y}-\frac{q_{3} q_{5}}{q_{1}^{2}} \frac{\partial q_{1}}{\partial y}-D\left(\frac{\partial^{2} q_{5}}{\partial x^{2}}-\frac{q_{5}}{q_{1}} \frac{\partial^{2} q_{1}}{\partial x^{2}}\right)-D\left(\frac{\partial^{2} q_{5}}{\partial y^{2}}-\frac{q_{5}}{q_{1}} \frac{\partial^{2} q_{1}}{\partial y^{2}}\right)=0
\end{gathered}
$$

Nótese que en el conjunto de ecuaciones (11) se encuentra incluido el término relacionado con la fuente de calor $\dot{q}$. La aproximación utilizada para modelar este término en un espacio discreto resulta ser:

$$
\begin{aligned}
& Q=\dot{m} \Delta h=\rho u A \Delta h \\
& q=\rho \Delta h
\end{aligned}
$$

Siendo $Q$ el flujo de calor, $\dot{m}$ el flujo de masa y $\Delta h$ la variación de la entalpía en el espacio diferencial, produciéndose un flujo de calor unitario $q$. Un modelo adecuado para evaluar la adición de calor en un gas perfecto es a través de la variación de la entalpía $h$. En un sistema donde la presión es esencialmente constante, tal como se asume en cámaras de combustión con llama difusiva, tal variable se expresa comúnmente como $h=c_{p}(T) T$, o $h \approx \bar{c}_{p}(T) T$, y así:

$$
q=\rho \bar{c}_{p} \Delta T
$$

De esta forma el término no homogéneo resulta expresado en función de la temperatura, $q=q(\rho, T)$, y ya que $T=T(f)$, entonces el calor añadido se puede expresar también como $q=q(\rho, f)$, dado que esta variable es una función de la fracción de mezcla.

Es así como el modelo propuesto resuelve, de forma acoplada, el campo de temperaturas a través de la fracción de mezcla y a partir de este campo de temperaturas se predice la tasa necesaria de calor añadido por combustión para llevar el sistema a tal estado.

\section{MODELO NUMÉRICO Y COMPUTACIONAL}

\section{Aproximación Numérica}

Para la solución del modelo matemático presentado, se hace uso de una formulación de primer orden de diferencias finitas para la aproximación de las derivadas parciales en el sistema (11). Esta aproximación genera un sistema explícito de ecuaciones en diferencias que se 
resuelve a través de un esquema de cálculo iterativo. La aproximación por diferencias finitas para las derivadas parciales [27] se formula como:

$$
\begin{gathered}
\frac{\partial q_{n}}{\partial t}=\frac{q_{n i, j}^{k+1}-q_{n i, j}^{k}}{K} \\
\frac{\partial q_{n}}{\partial x}=\frac{q_{n i, j}^{k}-q_{n i-1, j}^{k}}{h_{x}} \\
\frac{\partial q_{n}}{\partial y}=\frac{q_{n i, j+1}^{k}-q_{n i, j-1}^{k}}{2 h_{y}}
\end{gathered}
$$

En la notación para la variable discretizada $q^{k}{ }_{n i, j}$ los índices $i, j$ designan la posición de los nodos en los ejes $x$ e $y$ respectivamente y el superíndice $k$ representa el punto en el tiempo. Igualmente, $K=\Delta t$ es el valor del intervalo de tiempo discretizado, $h_{x}=\Delta x$ es la distancia entre nodos en el eje $x$ y $h_{y}=\Delta y$ es la distancia entre nodos en el eje $y$. La elección del tipo de diferencia finita a utilizar se formuló deseando obtener una relación conveniente entre el modelo, las condiciones de frontera y el sistema iterativo de cálculo. Así, todo término de derivada temporal se reemplaza por una diferencia hacia adelante para poder construir un sistema explícito donde los valores de las variables primitivas en un instante discreto de tiempo dependan únicamente de los valores puntuales del paso de tiempo inmediatamente anterior. Las derivadas en la dirección $y$ se reemplazan por aproximaciones en diferencias centrales para retener la influencia de las condiciones de frontera en $y=0$ e $y=L_{y}$.

Por último, en el caso de las derivadas en la dirección $x$ se toman diferencias hacia atrás para que la frontera $x=L_{x}$ adopte valores como resultado de la simulación y no sea necesario establecer allí una condición de frontera. La elección de diferentes tipos de diferencias en un mismo modelo naturalmente implicó la presencia de diferentes tasas de convergencia de solución en cada dirección, lo cual, aun cuando fue un aspecto a considerar en el algoritmo de solución, no afectó la precisión del mismo, una vez seleccionados los espaciamientos apropiados para el modelo. En general, en el modelo numérico propuesto, una variable primitiva $q^{k+1}$ es función de los valores de las variables primitivas en el instante de tiempo discreto inmediatamente anterior $k$, permitiendo formular un esquema de cálculo iterativo que marcha en el tiempo:

$$
\begin{aligned}
& q_{1 i, j}^{k+1}=f\left(K_{x}, K_{y}, q_{1 i, j}^{k}, q_{2 i, j}^{k}, q_{3 i, j}^{k}\right) \\
& q_{2 i, j}^{k+1}=f\left(K_{x}, K_{y}, q_{1 i, j}^{k}, q_{2 i, j}^{k}, q_{3 i, j}^{k}, q_{4 i, j}^{k}\right) \\
& q_{3 i, j}^{k+1}=f\left(K_{x}, K_{y}, q_{1 i, j}^{k}, q_{2 i, j}^{k}, q_{3 i, j}^{k}, q_{4 i, j}^{k}\right) \\
& q_{5 i, j}^{k+1}=f\left(K_{x}, K_{y}, q_{1 i, j}^{k}, q_{2 i, j}^{k}, q_{3 i, j}^{k}, q_{5 i, j}^{k}\right)
\end{aligned}
$$

Cualquier sistema de cálculo numérico explícito e iterativo que surja de la discretización del sistema de ecuaciones en derivadas parciales de característica hiperbólica definido en el conjunto de ecuaciones (11), está altamente condicionado al tamaño del intervalo de tiempo $K$ y a su relación con el tamaño de la discretización espacial $h_{x}$, según el número de Courant definido como $K / h_{x}$. Como principio general, este número debe ser menor a la unidad para garantizar la convergencia de la solución [28].

Aun cuando las formulaciones para flujos totalmente compresibles, como la adoptada en el presente trabajo, son apropiadas para el tratamiento de problemas en amplios regímenes de flujo, no resultan muy adecuadas en flujos de bajo número de Mach [29], esencialmente por el costo computacional que se deriva de la necesidad de usar intervalos de tiempo discreto muy reducidos con el fin de garantizar convergencia en las soluciones [17]. Así, la restricción numérica del paso de tiempo $K=\Delta t$ se puede expresar, mediante las condiciones CFL (Courant-Friedrichs-Lewy) [17, 28-29], para un caso bidimensional, como:

$$
K=\Delta t \leq C F L^{*} \min \left\{\frac{\Delta x}{|u+c|} ; \frac{\Delta y}{|v+c|}\right\}
$$

Donde CFL es un factor que depende de las propiedades de estabilidad del modelo y es de orden 1 . Esta condición se deriva de la idea que una onda acústica, que viaja a la velocidad del sonido $c$, sólo puede atravesar un dominio discreto de tamaño $\Delta x$ y que se mueve a una velocidad promedio $u$, si el tiempo discreto en que lo hace es menor que $\Delta t \leq \Delta x / \mid u+c$. De lo contrario la onda terminará difundiéndose en el dominio discreto y no lo atravesará como debería ocurrir físicamente.

A partir de las ecuaciones (4) y (9) se ha formulado un sistema expresado en variables normalizadas para garantizar dentro de los cálculos numéricos un peso relativo similar para cada variable. Con el fin de mejorar las condiciones de estabilización de la solución, las variables fueron normalizadas como: $\rho^{*}=\rho / \rho_{m}, u^{*}=u / c_{m}, v^{*}=v / c, p^{*}=p /$ $\left(\rho_{m} c_{m}\right), x^{*}=x / x_{m}, y^{*}=y / x_{m}$ y $t^{*}=c_{m} t / x_{m}$. En estas nuevas variables adimensionales, el subíndice $m$ se refiere a un valor máximo definido de antemano para la correspondiente variable y el superíndice * denota la variable normalizada. En particular, para los casos simulados, se tiene que: $\rho_{m}=10 \mathrm{~kg} / \mathrm{m}^{3}, x_{m}=0.5 m$ y $c_{m}=800 \mathrm{~m} / \mathrm{s}$. Estos valores corresponden a la mayor longitud en el dominio, y a los máximos valores de densidad y temperatura ${ }^{3}$ de las tablas de equilibrio químico obtenidas para una mezcla gaseosa

\footnotetext{
3 Con el fin de estimar la velocidad del sonido a través de la expresión $c_{m}=\sqrt{\gamma R T}$
} 
$\mathrm{C}_{3} \mathrm{H}_{8}$-Aire. El proceso de normalización inherentemente aumenta el costo computacional de la simulación, debido a que produce un sistema de ecuaciones con números de Courant normalizados, los cuales se ven amplificados por el factor $c_{m}$ (velocidad del sonido), tal como se define en (19).

$$
\frac{K^{*}}{h_{x}^{*}}=\frac{\Delta t^{*}}{h_{x}^{*}}=\frac{c_{m} \Delta t}{x_{m} \Delta x / x_{m}}=c_{m} \frac{K}{h_{x}}
$$

Es así como de forma implícita aumenta el requerimiento para el paso de tiempo $K$, si se desea mantener un mismo paso espacial $h_{x}$

\section{Condiciones iniciales y de frontera}

El dominio de simulación lo constituye un sistema bidimensional en el espacio más la variable temporal, con fronteras $\left[0, L_{x}\right],\left[0, L_{y}\right]$ y $\left[0, T_{f}\right]$ para $(x, y, t)$, respectivamente. Las condiciones de frontera aplicadas en el modelo expuesto se pueden resumir así:

\section{Velocidad:}

- $u(x=0, y, t)=u_{0}+u_{1} \sin \left(2 f_{r} t\right)$. Constituye la excitación del sistema en la entrada, donde $u_{0}$ es la componente media y $u_{l} \sin \left(2 f_{r} t\right)$ la componente fluctuante, siendo $f_{r}$ la frecuencia de estimulación. Se hace uso de una excitación de tipo sinusoidal dado su amplio uso en estudios relacionados $[8,10,30]$.

- $u(x, y=0, t)=u\left(x, y=L_{y}, t\right)=0 . v(x, y=0, t)=v\left(x, y=L_{y}, t\right)=0$. Velocidad nula en las paredes.

Fracción de mezcla:

- $f\left(x=L_{x}, y, t\right)=f(x, y=0, t)=f(x, y=L y, t)=0$, se plantea que en las paredes y en la salida del flujo existen únicamente productos de combustión.

- $f\left(x=0, y_{\text {boquilla }}, t\right)=1$, en la entrada de combustible (Boquilla).

- $f\left(x=0, y_{\text {aire, }}, t\right)=0$, en la entrada de aire.

Presión en la entrada del ducto:

- $\quad p(x=0, y, t)=1,45 \mathrm{MPa}$. Presión nominal alcanzada luego del paso del aire por el compresor en una turbina a gas.

Un aspecto a considerar en el planteamiento de las condiciones de frontera fue la eventual reflexión de ondas debido a la imposición de condiciones de frontera fijas [30]. Una onda generada físicamente dentro del propio modelo puede viajar por el dominio hasta encontrarse con una condición de frontera numérica (no física), por ejemplo una condición de presión o velocidad fija en la frontera de salida (ver Figura 3). Al encontrarse esta onda con un valor fijo en una frontera dada, este valor actuará como nodo de reflexión y la onda "reingresará" al dominio. Este fenómeno es propio del modelo numérico, pero evidentemente no se relaciona con el fenómeno físico. Para superar tal inconveniente existen tratamientos especiales para este tipo de fronteras, tales como condiciones de frontera características (Euler Characteristic Boundary Conditions ECBC) [2]. En el presente trabajo, dada la formulación escogida para las diferencias finitas en la dirección $x$, se evitó esta situación, pues los nodos de la frontera $x=L_{x}$ no eran nodos de reflexión al no prefijarse ninguna condición en éstos, de manera que las ondas "atraviesan" esta frontera sin ser reflejadas numéricamente en el dominio.

\section{Monitores de convergencia y estabilidad numérica}

Con el objeto de medir la velocidad con la cual el algoritmo alcanzaba un estado dado y evaluar la convergencia y exactitud general del modelo, se recurrió a la métrica $R_{n}$ dada por la siguiente relación [11]:

$$
R_{n}=\sqrt{\frac{\sum_{i=1}^{M_{x}} \sum_{j=1}^{M_{y}}\left(q_{n}(i, j, k+1)-q_{n}(i, j, k)\right)^{2}}{M_{x} M_{y}}}
$$

Esta expresión se evaluó para cada una de las variables primitivas $q_{n}$, con $n=1,2,3,4,5$, para los nodos $(i, j)$ en el dominio $(x, y)$. En la ecuación (20), $M_{x}$ corresponde al número de nodos del dominio en el eje $x$ y $M_{y}$ el número de nodos del dominio en el eje $y$. Con el fin de incrementar la estabilidad del método, se empleó un esquema de relajación numérica como el expuesto en la referencia [31]. El esquema de relajación utilizado es:

$$
q_{i, j}^{*}=(1-4 \xi) q_{i, j}+\xi\left(q_{i-1, j}+q_{i+1, j}+q_{i, j-1}+q_{i, j-1}\right)
$$

Siendo $\xi$ un pequeño factor, idealmente cero. El objetivo de usar factores de relajación $\xi$ es el de promediar levemente las soluciones a partir de los valores de la función en sus vecinos. En la Figura 6 se presenta el diagrama de pseudocódigo del esquema de cálculo iterativo planteado para la solución numérica del modelo propuesto.

\section{Solución del modelo de combustión en estado estable} Las derivadas temporales del sistema (11) son cero en estado estable. En consecuencia, para las ecuaciones en diferencias se puede afirmar que en estado estable $q_{n i, j}^{k+1}=q_{n i, j}^{k}$, lo que permitió construir un esquema de solución de marcha en el tiempo en el que la convergencia se obtiene cuando la solución de una iteración no se diferencia de la obtenida en la iteración anterior, independiente de la tolerancia fijada para los monitores de convergencia. Por tal motivo se requirió la definición de un tiempo de convergencia, arbitrario inicialmente, y una discretización temporal. En 
este caso se definió como tiempo de simulación $T_{f}=1 e-6$ $\mathrm{seg}$, dividido en 700 segmentos. Además, para un dominio con $M_{x}=120$ divisiones en el eje $x$ y $M_{y}=40$ divisiones en el eje $y$, se obtuvieron números de Courant normalizados de $K_{x}^{*}=2.742 e-4$ y $K_{y}^{*}=4.571 e-4\left(\operatorname{con} c_{m}=800 m / s\right)$. Así, la primera aproximación consistió en resolver un sistema bidimensional con las siguientes características:

- Combustible: $\mathrm{C}_{3} \mathrm{H}_{8}$; Oxidante: Aire $\left(79 \% \mathrm{~N}_{2}-21 \% \mathrm{O}_{2}\right)$

- Velocidad de entrada de aire $u_{0}=0,30 \mathrm{~m} / \mathrm{s}$. Velocidad de entrada de combustible $u_{0}=0,54 \mathrm{~m} / \mathrm{s}, u_{1}=0$.

- El coeficiente binario de difusión fue estimado a través de la aproximación de Reid [18] para una mezcla binaria $\mathrm{C}_{3} \mathrm{H}_{8}$-Aire, obteniéndose un valor de $D=2,2242 \times 10^{-5} \mathrm{~m}^{2} / \mathrm{s}$.

El algoritmo se implementó en forma computacional empleando MATLAB y FORTRAN como lenguajes de programación, en una máquina con arquitectura X86 a 32 bits. Para un nivel de tolerancia preestablecido de $T O L=0,0005$, los monitores de convergencia de cada variable primitiva evolucionaron como se muestra en la Figura 7. Esta simulación tardó aproximadamente $20 \mathrm{seg}$. en la etapa de procesamiento para la máquina descrita.

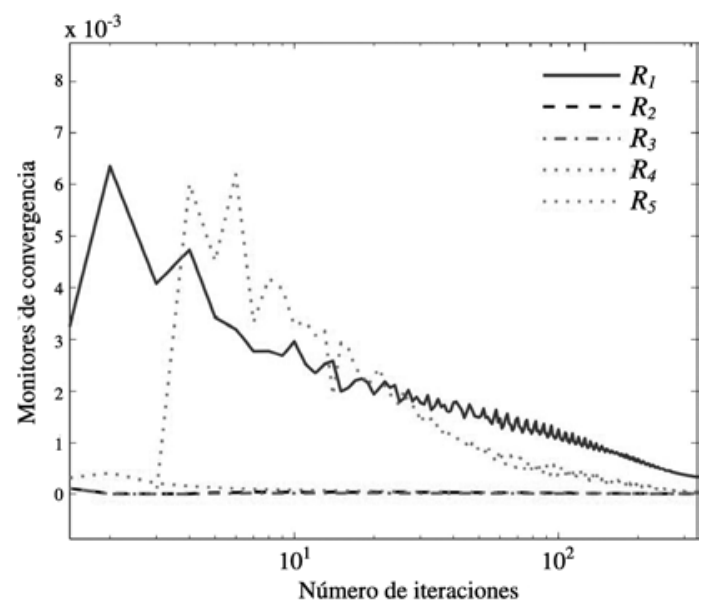

Figura 7. Monitores de convergencia $R_{n}$ para el modelo de combustión en estado estable (escala semilogarítmica).

Los monitores de convergencia se presentan usando una escala logarítmica en el eje del número de iteraciones, ya que en las primeras iteraciones existe una tendencia acelerada a la convergencia, la cual disminuye luego gradualmente. Los monitores de más lenta convergencia resultan ser las variables primitivas asociadas a la densidad y presión, como es de esperarse en los flujos compresibles [24].

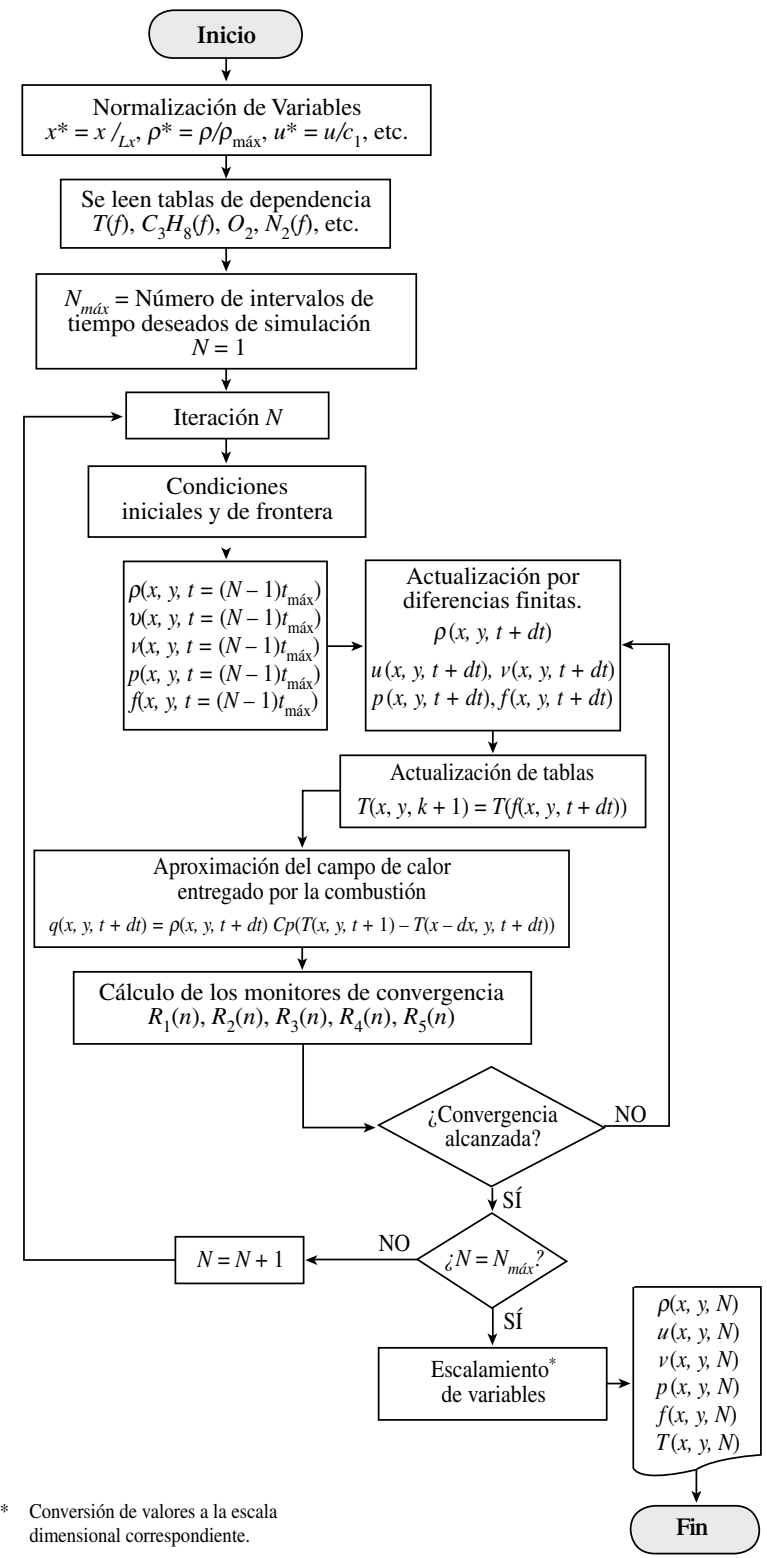

Figura 6. Pseudocódigo del algoritmo para la solución numérica del modelo propuesto.

Dada esta configuración, la convergencia numérica se alcanzó luego de 240 iteraciones. La Figura 8 presenta los resultados para el comportamiento de las variables en el sistema de combustión en estado estable luego de alcanzar la convergencia numérica.

Como se mencionaba anteriormente, la presente simulación corresponde a un sistema de combustión que presenta las características de una llama de difusión, así:

- Incremento de la temperatura a través de la llama debido al calor añadido al sistema por la combustión. 
- La presión para este tipo de proceso (una cámara con llama de difusión) esencialmente constante.

- La concentración del combustible disminuye, en tanto que la concentración de los productos aumenta.

- La densidad a través de la llama decrece.
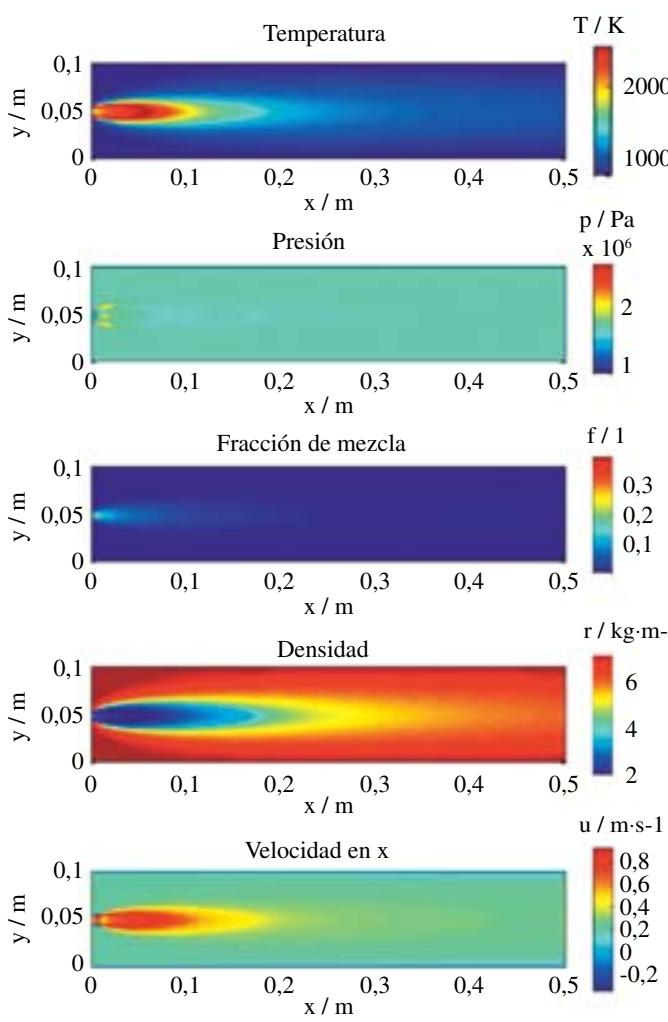

Figura 8. Perfiles de campo en estado estable, para el modelo propuesto. Temperatura, presión, fracción de mezcla, densidad y componente de velocidad en dirección $x$.

\section{Solución inestable del modelo de combustión}

Una vez establecida la implementación del sistema de combustión en estado estable, se pueden plantear varias estrategias para introducir la estimulación al sistema que induzca a la inestabilidad. Por ejemplo, se puede tomar una frontera modulada o bien en el tiempo o en la frecuencia como se expone en la referencia [8]. Otro método es la introducción al modelo estable de una perturbación acústica [32] con el fin de observar su interacción con el proceso de combustión, como es presentado en [5]. En el presente trabajo se eligió tomar una velocidad de entrada de aire modulada, definida por la función $u\left(x_{r e f}=0, y, t\right)=$ $0,3+0,03 \sin \left(2 \pi f_{r} t\right)$ con una frecuencia $f_{r}=500 \mathrm{~Hz}$, para un intervalo de tiempo simulado de $T_{f}=0,01 \mathrm{seg}$.

El problema de la simulación en el tiempo con una frontera modulada se plantea como la solución a $N$ subproblemas en estado estable, en el cual cada uno de ellos cubre un intervalo de tiempo $\Delta t=T / N$. Así, la solución global entonces estuvo constituida por la superposición en el tiempo de las $N$ simulaciones en estado estable, cada una de ellas con un determinado valor de estimulación en la velocidad de entrada. En las Figuras 9 y 10 se pueden observar la evolución de la fluctuación de la velocidad y presión respectivamente, obtenidas para un intervalo de tiempo característico.
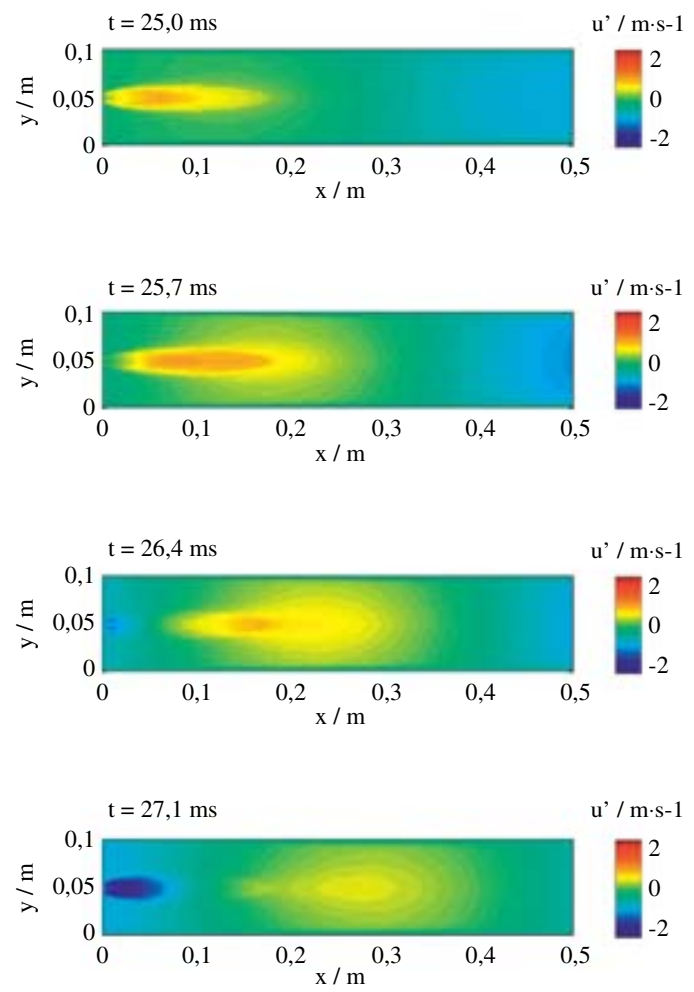

Figura 9. Evolución de la fluctuación de la velocidad axial en el tiempo.

El tiempo de procesamiento cuando el código se implementó en la máquina descrita y bajo los niveles de desratización mencionados se puede dividir en el procesamiento de la solución inicial, la cual tardó 20 segundos, y 3,5 segundos para cada una de las 699 subsecuentes iteraciones, es decir, un total de 2.466 seg. (41 minutos).

\section{ANÁLISIS DE RESULTADOS}

Una vez desarrollado un esquema de simulación del sistema de combustión, resulta importante hacer uso de éste para valorar respuestas de sistemas inestables simulados con el fin de reconocer y comparar parámetros, evaluando de paso la validez del modelo propuesto contra algún estudio disponible o información experimental. La función de transferencia de llama es un medio adecuado para encontrar una forma de comparación entre dos 
modelos de un mismo sistema de combustión inestable, dado que representa un modelo dinámico equivalente del comportamiento termoacústico del sistema. Así, es fundamental reconocer parámetros de funciones de transferencia de llama (como $n$ y $\tau$ en el modelo $n$ - $\tau$ ) si se quieren establecer estrategias de control dinámico de sistemas inestables o si se desea tratar el problema desde la ecuación termoacústica de onda.
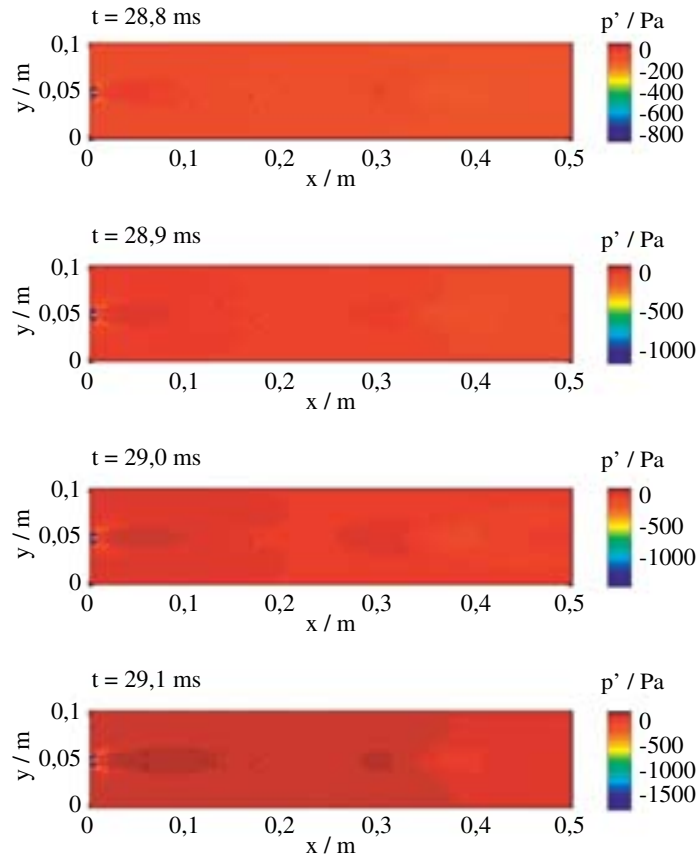

Figura 10. Evolución de la fluctuación de la presión en el tiempo.

En este sentido, como mecanismo de comparación, se eligió evaluar los parámetros obtenidos de la función de transferencia de llama del modelo $n-\tau$ de:

- Los resultados del modelo numérico y la información experimental disponible en la referencia [8].

- La solución basada en el esquema implementado en el presente trabajo de un modelo aproximado al desarrollado en la referencia [8].

El modelo propuesto en [8], considera las siguientes condiciones:

- Combustible $\rightarrow$ Propano $\left(\mathrm{C}_{3} \mathrm{H}_{8}\right)$.

- Relación global de aire/combustible real y estequiométrica $\emptyset=1,2$.

- Función de estimulación determinada por la modulación de la velocidad en la entrada, regida por la función $u(0, y, t)=4+0,4 \sin \left(2 \pi f_{r} t\right)$.

- Varias frecuencias de estimulación $f_{r}$, en el rango de $100 \mathrm{~Hz}$ a $600 \mathrm{~Hz}$.
- Un modelo basado en un dominio computacional representando un ducto quemador de aire/propano con varios orificios estabilizadores. En la Figura 11 se presenta un esquema parcial de esta configuración.

- En tal estudio se presenta una simulación numérica de flujo compresible basada en las ecuaciones de Navier-Stokes, incluyendo adicionalmente la química de reacción de la combustión.

- Adicionalmente, en [8] se hace referencia a un estudio experimental sobre este tipo de quemador con premezclado laminar, donde la llama es excitada por medio de un diafragma en la entrada, alterando de forma controlada la velocidad del flujo a la entrada del ducto de prueba, midiéndose luego la señal de velocidad de la llama a través de un anemómetro.

- Adicional a los perfiles del flujo, son extraídas de la simulación mediciones de la velocidad y tasa de calor fluctuantes en un punto dado.

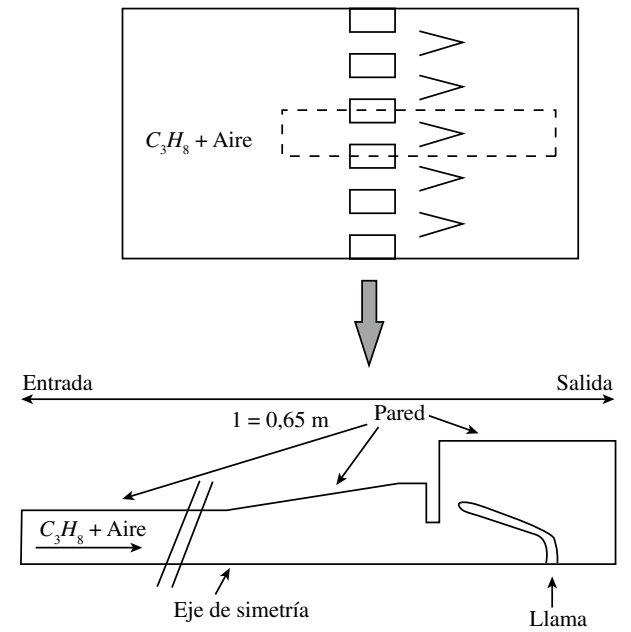

Figura 11. Características del dominio de prueba para el análisis desarrollado en la referencia [8].

A partir de la información presentada, se han determinado los parámetros de la función de transferencia de llama para el estudio presentado en [8], los cuales son ilustrados en la Figura 15.

Con el fin de comparar el modelo formulado en el presente trabajo, el mismo fue aplicado a un caso comparable al presentado en la referencia [8], contando con unas características de flujo y estimulación equivalentes y en el que se midieron los parámetros de la función de transferencia de llama. Es así como se definieron las siguientes características para el modelo desarrollado en el presente trabajo:

- Combustible: Propano $\left(\mathrm{C}_{3} \mathrm{H}_{8}\right)$.

- Relación global de mezcla $\emptyset=1,2$.

- Función de estimulación determinada por la relación $u(0, y, t)=4+0,4 \sin \left(2 \pi f_{r} t\right)$. 
- Frecuencia particular de estimulación $f_{r}=500 \mathrm{~Hz}$.

- $K_{x}=0,0013, K_{y}=0,0021, C F L=0,0033$.

- El dominio se representa en la Figura 12; la longitud de la cámara es $L_{x}=0,1 m$ y su dimensión transversal es $L_{y}=0,5 m$. En la Figura 12 se representa también el punto de medición de las señales de tasa de calor y velocidad, para la evaluación de la función de transferencia de llama (FTF).

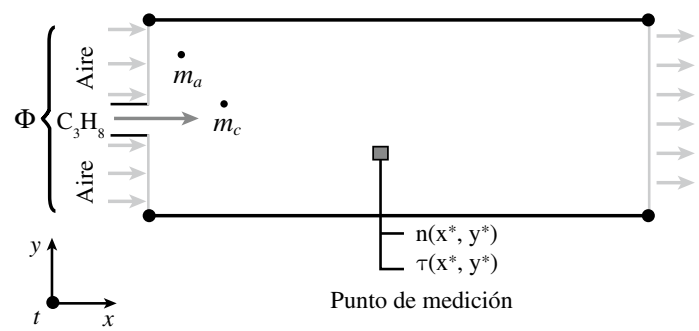

Figura 12. Punto de medición de señales de tasa de calor y velocidad para la evaluación de la FTF.

Como se mencionaba, a fin de conseguir convergencia numérica en el modelo, se utilizó un esquema de relajación, el cual en particular tomó los siguientes valores, para cada una de las variables primitivas: $\xi_{1}=0,002, \xi_{2}=0,002$, $\xi_{3}=0, \xi_{4}=0,005$ y $\xi_{5}=0,2$. Estos valores se determinaron durante la implementación computacional del modelo propuesto.

Adicionalmente, una vez definidas las dimensiones del dominio en términos de geometrías de entrada de aire y de combustible, se ajustaron los valores de velocidad de entrada de cada uno de los flujos, de manera que se obtuviera la relación global de mezcla definida anteriormente $(\varnothing=1,2)$. Los valores para las longitudes del dominio $L_{y}$ y las propiedades como densidad $\rho$, flujo másico $\dot{m}$ y velocidad $u$ en la dirección $x$, para cada una de las zonas de entrada tanto de aire como de combustible se presentan en la Tabla 1.

Tabla 1. Valores usados en el modelo para obtención de una relación aire-combustible de $A C=13$ $(\varnothing=1,2)$.

\begin{tabular}{|c|c|c|c|c|}
\hline Gas fluyendo & $\begin{array}{c}\boldsymbol{L}_{\mathbf{V}}[\mathbf{m}] \\
(\text { entrada) }\end{array}$ & $\rho\left[\mathbf{k g} / \mathbf{m}^{\mathbf{3}}\right]$ & $\boldsymbol{m}[\mathbf{k g} / \mathbf{s}]$ & $\boldsymbol{u}[\mathbf{m} / \mathbf{s}]$ \\
\hline Aire & 0,0974 & 7.188 & 2,8033 & 4 \\
\hline Combustible & 0,0050 & 3.786 & 0,2131 & 10 \\
\hline
\end{tabular}

Parámetros de la función de transferencia de llama La función de transferencia de llama (FTF) del modelo $n$ - $\tau$ es la relación entre las señales de velocidad fluctuante en un punto de referencia y la tasa de calor fluctuante en otro punto dado. Esto es equivalente a suponer que la tasa de calor fluctuante es proporcional a la fluctuación de la velocidad multiplicada por una cantidad $n$ con un retardo en las señales de $\tau$, dentro de la etapa de amplitud constante de las ondas. De esta forma, si en un punto particular se toma la función de velocidad y se hace un corrimiento en el tiempo de valor $\tau$, entonces las ondas normalizadas de tasa de calor y velocidad de entrada estarán en fase.

Para la función de transferencia de llama de un sistema de combustión con modulación de la velocidad en la posición de referencia $\left(x_{\text {rej }} y_{\text {ref }}\right)$ se puede enunciar según el modelo $n-\tau[9,33]$ que:

$$
\frac{\dot{q}_{1}(x, y, t)}{\dot{q}_{0}(x, y, t)}=n(x, y) \frac{\vec{u}_{1}\left(x_{r e f}, y_{r e f}, t-\tau\right) \cdot \vec{n}_{r e f}}{\vec{u}_{0}\left(x_{r e f}, y_{r e f}\right) \cdot \vec{n}_{r e f}}
$$

Tomando como punto de referencia la entrada del dominio $\left(x_{r e f}^{*}=0,0, y_{r e f}^{*}=0,25\right)$ y con el vector unitario de velocidad en la entrada alineado con el eje $x$, la expresión del índice de interacción se puede reducir a:

$$
\begin{aligned}
\frac{\dot{q}_{1}(x, y, t)}{\dot{q}_{0}(x, y, t)} & =n(x, y) \frac{u_{1}(0,0.25, t-\tau)}{u_{0}(0,0.25)} \\
& \therefore n(x, y)=\frac{u_{0} \dot{q}_{1}(x, y, t)}{u_{1}(t-\tau) \dot{q}_{0}(x, y, t)}
\end{aligned}
$$

Esta relación permite reconocer explícitamente las variables requeridas para determinar el factor de amplificación $n$ de la simulación, el cual es finalmente la variable adimensional con la que se realiza la comparación de resultados.

\section{Comparación de resultados}

Para llevar a cabo la comparación de resultados con los trabajos de referencia, se determinaron de la simulación computacional, y a partir de las curvas de velocidad y tasa fluctuante de calor, los parámetros $n$ y $\tau$ para un punto equivalente del dominio.

Para definir el punto de medición de las señales se tomó entonces como posición de referencia la coordenada normalizada $\left(x^{*}=0,0, y^{*}=0,25\right)$ y como punto de medición de la velocidad y la tasa de calor fluctuante la coordenada normalizada $\left(x^{*}=0,7, y^{*}=0,25\right)$. Así se garantizó que la distancia de separación entre estos dos puntos en el eje horizontal fuera de $0,35 \mathrm{~m}$, distancia equivalente a la utilizada en la referencia [8] para la separación entre la excitación y la llama inestable.

El retardo entre las ondas obtenido en el presente trabajo es de $\tau=0,45 \mathrm{~ms}$ (ver Figura 13), en tanto que en [8], para una frecuencia de $500 \mathrm{~Hz}$, este tiempo de retardo es de $\tau=0,5 \mathrm{~ms}$. El índice de interacción $n$ obtenido para 
el punto normalizado de medición $\left(x^{*}=0,7, y^{*}=0,25\right)$ es presentado en la Figura 14. En tal gráfico se aprecia que la tasa de calor de combustión normalizada obtenida presentó una amplitud de 0,4 , en tanto que la de velocidad en la entrada es de 0,1 , con lo que se obtuvo finalmente un índice de interacción de $n=4,4$.

Nuevamente, el resultado experimental de [8] para el índice de interacción es $n=4,0$. De esta forma los resultados para el índice de interacción y el tiempo de retardo obtenidos en el presente estudio mostraron coherencia con los resultados presentados por Nicoud [8]. Ambos estudios se han llevado a cabo con la misma modulación en la excitación de la velocidad de aire en la entrada y cubren dominios y relaciones de mezcla equivalentes.

Tasa de calor fluctuante y velocidad fluctuante normalizadas

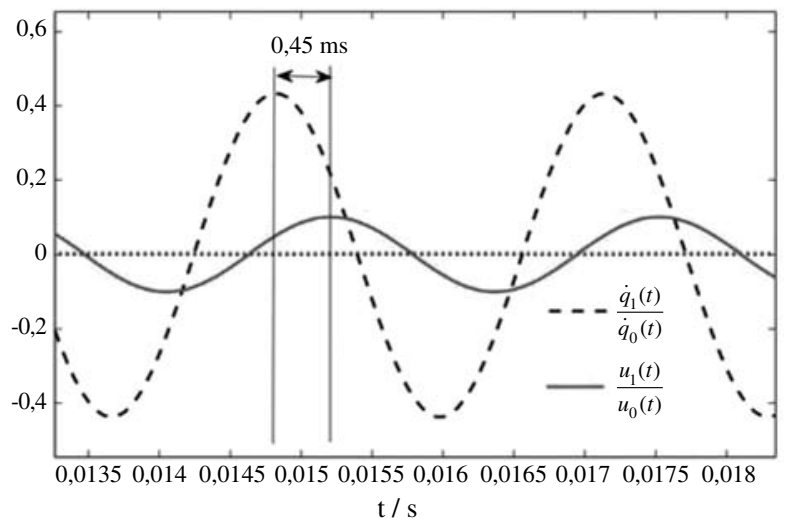

Figura 13. Retardo entre ondas $\tau$ obtenido para la coordenada normalizada $\left(x^{*}=0,7, y^{*}=0,25\right)$.

Tasa de calor fluctuante y velocidad fluctuante normalizadas

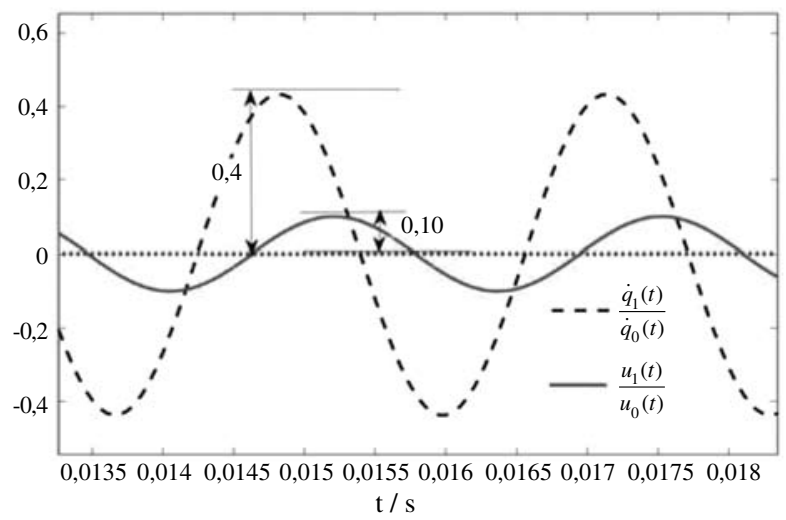

Figura 14. Índice de interacción $n$ obtenido para la coordenada normalizada $\left(x^{*}=0,7, y^{*}=0,25\right)$.

\section{ANÁLISIS EN MEZCLAS POBRES}

Es un fenómeno claramente reconocido a nivel práctico, que el uso de mezclas pobres en turbinas a gas, bien sea para disminución de consumo de combustible o para reducción de contaminantes, aumentan la sensibilidad del sistema a fenómenos de combustión inestable [7,34,35]. Con el fin de corroborar la coherencia del modelo propuesto, según esta clara tendencia en los sistemas reales, otra de las respuestas a comprobar desde el punto de vista numérico resulta ser la verificación del efecto de la inestabilidad en las mezclas pobres. De acuerdo a su definición, era apropiado evaluar este efecto a través del factor de amplificación $n$ de la función de transferencia de llama (FTF) en el modelo $n$ - $\tau$ [9]. De esta forma, deberían obtenerse factores de amplificación mayores en cuanto más pobre fuera la mezcla. Para una mezcla de gases reactantes se puede definir siempre la relación de equivalencia como:

$$
\phi=\frac{A C_{\text {stoic }}}{A C}
$$

Este es un parámetro que mide qué tan "pobre" es una mezcla en forma global. Así, para una relación de airecombustible pobre el valor de $\emptyset$ deberá ser menor que la unidad. En una mezcla de aire (compuesta por $21 \% \mathrm{O}_{2}$ y $\left.79 \% \mathrm{~N}_{2}\right)$ y propano $\left(\mathrm{C}_{3} \mathrm{H}_{8}\right)$, la relación aire/combustible $A C$ para una reacción estequiométrica es de $A C_{\text {stoic }}=15,6$, por lo que en una mezcla pobre $A C>15,6$.

Teniendo en cuenta esta definición, se plantearon 7 escenarios de simulación distintos, en los que gradualmente se hizo la mezcla más pobre ( $\varnothing$ cada vez menor), a través del aumento de la velocidad fluctuante de flujo de aire en la entrada en el dominio numérico, con $u(0, y, t)=u_{0}+0,1 u_{0} \sin (2 \pi 500 t)$, manteniendo el flujo de combustible constante. Por lo tanto, el objetivo fue determinar el factor de amplificación asociado a cada uno de los escenarios, con un par de puntos particulares. El factor de amplificación $n$ de una perturbación de velocidad aplicada en una posición de referencia es (reescribiendo la ecuación (23)):

$$
n(x, y)=\frac{\dot{q}_{1}(x, y, t) \vec{u}_{0}\left(x_{r e f}, y_{r e f}\right) \cdot \vec{n}_{r e f}}{\dot{q}_{0}(x, y, t) \vec{u}_{1}\left(x_{r e f}, y_{r e f}, t-\tau\right) \cdot \vec{n}_{r e f}}
$$

Este factor es función de la posición $n=n(x, y)$ donde se evalúa la tasa de calor añadida por combustión $q\left(x_{p}, y_{p}\right)$ $\mathrm{y}$, por tanto, en general, diferente en cada punto del dominio. En cualquier caso, la estimulación externa $u\left(x_{r e f} y_{\text {ref }}, t\right)$ se tomó como una fluctuación sinusoidal de la velocidad de entrada del aire. De esta forma, para evaluar el factor $n$ en algún punto en particular $n\left(x_{p}, y_{p}\right)$ basta con extraer de la simulación numérica la función de la tasa de calor fluctuante entregada por la combustión en el correspondiente punto. En este punto se utilizaron los siguientes parámetros de simulación:

- $K x=0,0013, K y=0,0021, M_{x}=120$ y $M_{y}=40$.

- $\xi_{1}=0,002, \xi_{2}=0,002, \xi_{3}=0, \xi_{4}=0,005$ y $\xi_{5}=0,2$. 
Para cada valor de velocidad probado, se generó entonces un flujo másico de aire que definió la relación global $\varnothing$ asociada a cada escenario. Las relaciones de velocidad y flujo másico de aire y la relación global $\varnothing$ de las mezclas obtenidas se presentan en la Tabla 2. En todos los casos la densidad del aire a la entrada se tomó como $\rho(0, y, t)=7.188 \mathrm{~kg} / \mathrm{m}^{3}$, con un flujo de combustible (por unidad de profundidad) de $m_{c}=0,1395 \mathrm{~kg} / \mathrm{s}$.

Tabla 2. Valores de flujos para los escenarios de prueba del modelo con mezclas pobres.

\begin{tabular}{|c|c|c|c|}
\hline Escenario & $\boldsymbol{u}_{\boldsymbol{o}}(\boldsymbol{0}, \boldsymbol{y}, \boldsymbol{t})[\mathbf{m} / \mathbf{s}]$ & $\boldsymbol{m}_{\boldsymbol{a}}[\mathbf{k g} / \mathbf{s m}]$ & $\boldsymbol{\emptyset}$ \\
\hline 1 & 4,75 & 3.107 & 0,70 \\
\hline 2 & 4,50 & 2.943 & 0,74 \\
\hline 3 & 4,25 & 2.780 & 0,78 \\
\hline 4 & 4,00 & 2.616 & 0,83 \\
\hline 5 & 3,75 & 2.453 & 0,89 \\
\hline 6 & 3,50 & 2.289 & 0,95 \\
\hline 7 & 3,25 & 2.126 & 1,02 \\
\hline
\end{tabular}

Los factores de amplificación se midieron usando la ecuación (25), para dos puntos particulares del dominio. Tales puntos, en coordenadas normalizadas, fueron $\left(x^{*}, y^{*}\right)=(0,15,0,25)$ y $\left.\left(x^{*}, y^{*}\right)=(0,35,0,25)\right)$. Estos puntos fueron relacionados con las respectivas relaciones de equivalencia. Los flujos de reactantes, dominio y los puntos de medición de las ondas de tasa de calor fluctuante se representan en la Figura 15.

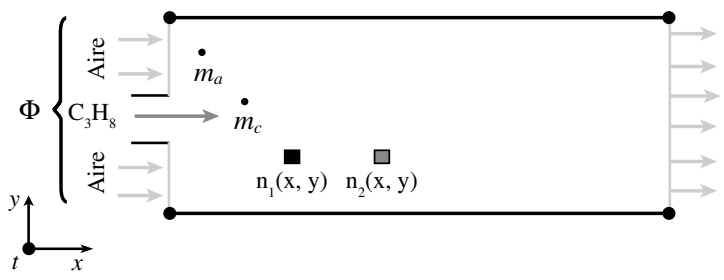

Figura 15. Representación esquemática del modelo para la evaluación del factor de amplificación $n$ en análisis de mezclas pobres.

Las respuestas del modelo propuesto en términos de la relación de equivalencia $\emptyset$ y el factor de amplificación $n$, para los escenarios con mezclas pobres se muestran en la Figura 16. Para ambos puntos de medición se encontró una fuerte relación al aumento del factor de amplificación con respecto a una disminución de la relación de equivalencia. Esto muestra que a nivel de la simulación numérica una mezcla pobre presenta una mayor tendencia a la amplificación de la onda de tasa de calor fluctuante, la cual es una fuente directa de combustión inestable.

\section{CONCLUSIONES}

Se ha formulado e implementado un modelo numérico con variables normalizadas, para un flujo compresible y de química de reacción para combustión en equilibrio, con el fin de obtener una aproximación de la descripción del fenómeno de combustión inestable en un dominio bidimensional. Se presenta explícitamente la formulación de un algoritmo de solución numérica de este modelo con un alto nivel de detalle.

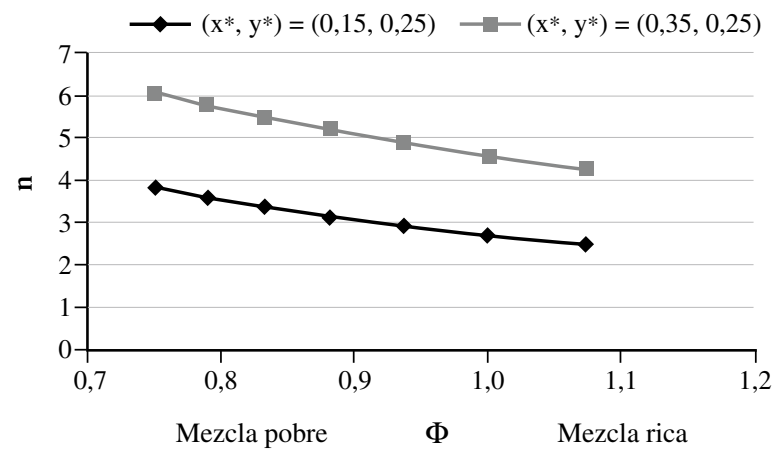

Figura 16. Variación del factor de amplificación para los escenarios de prueba con diferentes valores de $\varnothing$. Mezcla de $C_{3} H_{8}$ y aire.

El algoritmo desarrollado para resolver de manera explícita el sistema de ecuaciones formulado ha mostrado una eficiencia adecuada, dado el nivel de simplificación, en el cálculo y simulación del sistema base, permitiendo además incorporar el tratamiento de fronteras no-reflexivas y presentando un conveniente grado de flexibilidad para aplicarse a modelos más complejos. Sin embargo, el modelo construido y propuesto presenta una moderada dependencia de las condiciones iniciales. Esta formulación también permite la incorporación de una alta variedad de fronteras moduladas para la simulación de excitaciones a la entrada de un sistema equivalente al propuesto en el presente trabajo.

El modelo permitió además revelar el campo fluido interno de un proceso de combustión por difusión a través de la implementación de un código computacional compacto, desarrollado en una máquina de cómputo convencional. La implementación computacional se realizó usando dos tipos de lenguaje de programación: MATLAB y FORTRAN. El algoritmo presentó un buen desempeño computacional, en términos de tiempos de ejecución, con ambos lenguajes de programación, utilizando tanto el lenguaje interpretado (MATLAB) como el compilado (FORTRAN), en una máquina convencional de cómputo de arquitectura $\mathrm{x} 86$ a 32 bits. 
La formulación de un sistema con variables normalizadas con el propósito de garantizar un balance entre los aportes de cada una de las variables originales dentro del modelo numérico, independiente de su orden de magnitud, ha mostrado una excelente efectividad como mecanismo de compensación global de las variables en el modelo. No obstante, fue evidente que este proceso de normalización incrementó el costo computacional de la simulación al requerir un tamaño de malla menor para así obtener números de Courant que promovieran la convergencia de la solución y que al mismo tiempo garantizaran las condiciones CFL.

Con el objetivo de medir la coherencia de los resultados presentados con los de otro estudio disponible, se tomó como elemento de comparación la función de transferencia de llama (FTF) del modelo $n$ - $\tau$, la cual fue planteada como una forma compacta para modelar de forma general la dinámica del fenómeno de inestabilidad en combustión a través de los parámetros $n$ y $\tau$. Se ha obtenido un excelente grado de aproximación, dadas las simplificaciones incorporadas, entre la simulación bidimensional y el estudio experimental con el que se ha comparado.

Igualmente, haciendo uso de la simulación desarrollada, se ha mostrado un análisis del efecto de la combustión inestable de mezclas pobres de $C_{3} H_{8}$ /aire. Usando el índice de amplificación de la función de transferencia de llama, se valoró la hipótesis de mayor sensibilidad a efectos de combustión inestable en mezclas pobres. Los resultados del modelo desarrollado mostraron una respuesta totalmente acorde con tal hipótesis.

\section{AGRADECIMIENTOS}

Los autores desean agradecer al Instituto Colombiano para el desarrollo de la Ciencia y la Tecnología COLCIENCIAS y a la Universidad Nacional de Colombia, los cuales dentro del marco del programa conjunto "Jóvenes Investigadores e Innovadores Año 2007” dieron su apoyo a la presente investigación.

\section{REFERENCIAS}

[1] J. Rayleigh. "The Nature of Sound". Dover, New York, USA. 1978.

[2] T. Poinsot and D. Veynante. "Theoretical and Numerical Combustion”. Edwards. Philadelphia, PA, USA. 2001.

[3] X. Wu, M. Wang, P. Moin and N. Peters. "Combustion instability due to the nonlinear interaction between sound and flame". Journal of Fluid Mechanics, Vol. 497, pp. 23-54. 2003.
[4] H.J. Viljoen and L. Boshoff-Mostert. "Analysis of combustion-driven acoustics". Chemical Engineering Science. Vol. 53, pp. 1679-1687. May 01, 1998.

[5] A. Laverdant and D. Thevhenin. "Interaction of a gaussian acoustic wave with a turbulent premixed flame". Combustion and Flame. Vol. 134, pp. 11-19. 2003.

[6] A.H. Lefrevbe. "Gas Turbine Combustion". Taylor and Francis. Second Edition. 1999.

[7] C. Martin, L. Benoit, Y. Sommerer, F. Nicoud and T.J. Poinsot. "Large-eddy simulation and acoustic analysis of a swirled staged turbulent combustor". AIAA (American Institute of Aeronautics and Astronautics) Journal. Vol. $44 \mathrm{~N}^{\circ}$ 4, pp. 741-750. 2006.

[8] A. Kaufman, F. Nicoud and T. Poinsot. "Flow Forcing Techniques for Numerical Simulation of Combustion Instabilities". Combustion and Flame. Vol. 131, pp. 371-385. 2002.

[9] L. Benoit and F. Nicoud. "Numerical assessment of thermo-acoustic instabilities in gas turbines". International Journal for Numerical Methods in Fluids. Vol. 47, Issues 8-9, pp. 849-855. 2005.

[10] B. Varoquié, J.P. Légier, F. Lacas, D. Veynante and T. Poinsot. "Experimental analysis and large eddy simulation to determine the response of nonpremixed flames submitted to acoustic forcing". Proceedings of the Combustion Institute. Vol. 29, Issue 2, pp. 1965-1970. 2002.

[11] R. Bhaskaran and L. Collins. "Introduction to CFD basics". Cornell University - Sibley School of Mechanical and Aerospace Engineering. 2002.

[12] C. Pera and J. Reveillon. "Direct Numerical Simulation of spray flame/acoustic interaction". Proceedings of the Combustion Institute. Vol. 31, pp. 2283-2290. 2007.

[13] F. di Mare, W.P. Jones and K.R. Menzies. "Large Eddy Simulation of a model gas turbine combustor". Combustion and Flame. Vol. 137, pp. 278-294. 2004.

[14] G. Staffelbach, L.Y.M. Gicquel, G. Boudeir and T. Poinsot. "Large Eddy Simulation of self excited azimuthal modes in annular combustors". Proceedings of the Combustion Institute. Vol. 32, Issue 2, pp. 2909-2916. 2009.

[15] F. Flemming, A. Sadiki and J. Janicka. "Investigation of combustion noise using a LES/CAA hybrid approach". Proceedings of the Combustion Institute. Vol. 32, Issue 2, pp. 3189-3196. January 2007.

[16] J. Janicka and A. Sadiki. "Large Eddy Simulation of Turbulent combustion systems". Proceedings of the Combustion Institute. Vol. 30, Issue 1, pp. 537547. January 2005. 
[17] Y. Wang and A. Trouvé. "Artificial acoustics stiffness reduction in fully compressible, direct numerical simulation of combustion". Combustion Theory and Modelling. Vol. 8, Issue 3, pp. 633660. September 2004.

[18] S. Turns. "An introduction to combustion, concepts and applications". McGraw-Hill. New York, USA. 2000.

[19] Y.Y. Lee and S.B. Pope. "Nonpremixed turbulent reacting flow near extinction". Combustion and Flame. Vol. 101, pp. 501-528. 1995.

[20] L. Vervisch. "Using numerics to help the understanding of non-premixed turbulent flames". Proceedings of the Combustion Institute. Vol. 28, Issue 1, pp. 11-24. 2000.

[21] C.K. Westbrook, Y. Mizobuchi, T.J. Poinsot, P.J. Smith and J. Marnatz. "Computational Combustion". Proceeding of the Combustion Institute. Vol. 30, Issue 1, pp. 125-157. January 2005.

[22] H. Lomax, T.H. Pulliam and D.W. Zingg. "Fundamentals of Computational Fluid Dynamics". NASA Ames Research Center - University of Toronto Institute for Aerospace Studies. August 05, 1999.

[23] M. Gineste. "Simulation of Thermoacoustics with Discontinous Galerkin Method". Kongens Lyngby. 2006.

[24] S. W. Rienstra and A. Hirschberg. "An Introduction to Acoustics". Eindhoven University of Technology. 2006.

[25] A. Fichera, C. Losenno and A. Pagano. "Experimental analysis of thermo-acoustic combustion instability". Applied Energy. Vol. 70 $\mathrm{N}^{\circ}$ 2, pp. 179-191. October 2001.

[26] B. Michaelis and B. Rogg. "FEM-Simulation of laminar flame propagation. I:Two-dimensional flames". Journal of computational physics. Vol. 196, Issue 2, pp. 417-447. May 20, 2004.
[27] J.R. LeVeque. "Finite Difference Methods for Dierential Equations". University of Washington. 2005.

[28] I.J. Keshtiban, F. Belblidia and M.F. Webster. "Compressible flow solvers for low Mach number flows-a review". Technical Report CSR2. Institute of Non-Newtonian Fluid Mechanics. University of Wales. Swansea, UK. 2004.

[29] T. Poinsot, S. Candel and A Trouvé. "Applications to direct numerical simulation to premixed turbulent combustion". Progress in Energy and Combustion Science. Vol. 21, Issue 6, pp. 531-576. 1995.

[30] F. Nicoud and T. Poinsot. "Boundary conditions for compressible unsteady flows". In Artificial Boundary Conditions at Interfaces. L. Halpern, F. Nataf and L. Tourrette, Editors. Nova Science Publishers. New York, USA. 2001.

[31] D. Thibaut and S. Candel. "Numerical study of unsteady turbulent premixed combustion: Application to flashback simulation". Combustion and Flame. Vol. 113, Issues 1-2, pp. 53-65. April 1998.

[32] M.P. Juniper, L.K.P. Li and J.W. Nichols. "Forcing of self-excited round jet diffusion flames". Proceedings of the Combustion Institute. Vol. 32, Issue 1, pp. 1191-1198. 2009.

[33] M. Fleifil, A.M. Annaswamy, Z.A. Ghoneim and A.F. Ghoniem. "Response of a laminar premixed flame to flow oscillations: A kinematic model and thermoacustic instability results". Combustion and Flame. Vol. 106, pp. 487-510. 1996.

[34] J.P. Moeck, M. Oevermann, R. Klein, C.O. Paschereit and H. Schmidt. "A two-way coupling for modelling thermoacoustic instabilities in a flat flame Rijke tube". Proceedings of the Combustion Institute. Vol. 32, Issue 1, pp. 1199-1207. 2009.

[35] D. Fritsche, M. Füri and K. Boulouchos. "An experimental investigation of thermoacoustic instabilities in a premixed swirl-stabilized flame". Combustion and Flame. Vol. 151, Issues 1-2, pp. 29-36. October 2007. 